Research Article

\title{
Some New Connection Relations Related to Classical Orthogonal Polynomials
}

\author{
Wathek Chammam $\mathbb{D}^{1,2,3}$ and Wasim Ul-Haq $\mathbb{D}^{1,4}$ \\ ${ }^{1}$ Department of Mathematics, College of Science Al-Zulf, Majmaah University, P.O. Box 66, Al-Majmahh 11952, Saudi Arabia \\ ${ }^{2}$ Mathematics and Applications Laboratory, Faculty of Science, Gabés University, 6072 Gabès, Tunisia \\ ${ }^{3}$ Department of Electro Mechanics, Higher Institute of Industrial Systems of Gabés, 6072 Gabès, Tunisia \\ ${ }^{4}$ Department of Mathematics, Abbottabad University of Science and Technology, Abbottabad 22010, Pakistan
}

Correspondence should be addressed to Wathek Chammam; w.chammam@mu.edu.sa

Received 26 April 2020; Accepted 3 June 2020; Published 24 June 2020

Academic Editor: Basil K. Papadopoulos

Copyright ( 2020 Wathek Chammam and Wasim Ul-Haq. This is an open access article distributed under the Creative Commons Attribution License, which permits unrestricted use, distribution, and reproduction in any medium, provided the original work is properly cited.

In this paper, we deal with a problem of positivity of linear functionals in the linear space $\mathbb{P}$ of polynomials in one variable with complex coefficients. Some new results of connection relations between the corresponding sequences of monic orthogonal polynomials of classical character are established.

\section{Introduction}

The criterion of positivity is important in special functions theory, such as approximation theory, moment problem, and orthogonal polynomials and applications, among others, because the solutions of many problems depend on the determination of when a specific function is positive or nonnegative. For example, several authors have focused on the study of linear positive operators and their applications in problems and inequalities. Here, we can mention Aloui [1, 2], Bennett [3], and others. Also, we quote the work of Chen et al. [4] in the total positivity of Riordan arrays.

The linear functional $\mathcal{U}$ is positive definite if and only if $\Delta_{n}(\mathscr{U})>0$, for every integer $n \geq 0$, where $\Delta_{n}(\mathcal{U})$ is the Hankel determinants of order $n$ of $\mathscr{U}$ (see [5]). In order to provide a construction process of positive-definite linear functional from positive-definite linear data, Sfaxi has proved a new construction process and he also gave an example a second-order positive-definite linear functional from a Laguerre positive-definite linear functional [6].

The outline of this paper is as follows. In Section 2, we introduce the basic background and notations to be used throughout the paper. In Section 3, we present our main results. Indeed, using Oppenheim's inequality we prove that, for any pair $(\mu, c) \in] 0,+\infty[\times \mathbb{R}$ and any positive-definite linear functional $\mathcal{U}$, the linear functional $\mathscr{U}(\mu, c)$ given by $(\mu-1) \mathcal{U}(\mu, c)-(x-c)(\mathcal{U}(\mu, c))^{\prime}=\mu \mathcal{U}$ is also positive definite. Finally, some new results of connection relations between the corresponding sequences of monic classical orthogonal polynomials are presented.

\section{Orthogonality and Positive-Definite Linear Functional Character}

Let $\mathbb{P}$ be the linear space of polynomials in one variable with complex coefficients and $\mathbb{P}^{\prime}$ its algebraic dual space. We denote by $\langle\mathscr{U}, p\rangle$ the action of $\mathscr{U} \in \mathbb{P}^{\prime}$ on $p \in \mathbb{P}$ and by $(\mathcal{U})_{n}:=\left\langle\mathscr{U}, x^{n}\right\rangle, n \geq 0$, the sequence of moments of $\mathscr{U}$ with respect to the polynomial sequence $\left\{x^{n}\right\}_{n \geq 0}$. Let us define the following operations in $\mathbb{P}^{\prime}$. For any linear functionals $\mathscr{U}$ and $\mathscr{V}$, any polynomial $q$, and any $(a, b, c) \in \mathbb{C}^{*} \times \mathbb{C}^{2}$, let $D \mathscr{U}=\mathscr{U}^{\prime}, q \mathcal{U},(x-c)^{-1} \mathcal{U}, \tau_{-b} \mathcal{U}$, $h_{a} \mathscr{U}$, and $\mathscr{U} \mathscr{V}$ be the linear functionals defined by duality (see $[7,8])$ : 


$$
\begin{aligned}
&\left\langle\mathcal{U}^{\prime}, p\right\rangle:=-\left\langle\mathcal{U}, p^{\prime}\right\rangle, \\
&\langle q \mathcal{U}, p\rangle:=\langle\mathcal{U}, q p\rangle, \\
&\left\langle(x-c)^{-1} \mathscr{U}, p\right\rangle:=\left\langle\mathcal{U}, \theta_{c} p\right\rangle=\left\langle\mathcal{U}, \frac{p(x)-p(c)}{x-c}\right\rangle, \\
&\left\langle\tau_{-b} \mathcal{U}, p\right\rangle:=\left\langle\mathcal{U}, \tau_{b} p\right\rangle=\langle\mathcal{U}, p(x-b)\rangle, \\
&\left\langle h_{a} \mathcal{U}, p\right\rangle:=\left\langle\mathcal{U}, h_{a} p\right\rangle=\langle\mathcal{U}, p(a x)\rangle, \\
&\langle\mathcal{U}, p\rangle:=\langle\mathcal{U}, \mathscr{V} p\rangle, \quad p \in \mathbb{P},
\end{aligned}
$$

where the right-multiplication of $\mathscr{V}$ by $p$ is a polynomial given by

$$
(\mathscr{V} p)(x):=\left\langle\mathscr{V}_{y}, \frac{x p(x)-y p(y)}{x-y}\right\rangle, \quad p \in \mathbb{P} .
$$

For any $c \in \mathbb{C},(x-c)^{-1}(x-c) \mathcal{U}=\mathscr{U}-(\mathscr{U})_{0} \delta_{c}$, where $\delta_{c}$ is the Dirac functional defined by $\left\langle\delta_{c}, p\right\rangle=p(c), p \in \mathbb{P}$.

Notice that if $\mathscr{U} \in \mathbb{P}^{\prime}$ is such that $\mathcal{U}^{\prime}=0$, then $\mathscr{U}=0$.

A linear functional $\mathscr{U}$ is said to be quasi-definite (regular) if we can associate with it a monic polynomial sequence (MPS) $\left\{B_{n}\right\}_{n \geq 0}$ with $\operatorname{deg} B_{n}=n, n \geq 0$, such that

$$
\left\langle\mathcal{U}, B_{n} B_{m}\right\rangle=r_{n} \delta_{n, m}, \quad m, n \geq 0 \text {, and where }{ }_{n} \neq 0, n \geq 0,
$$

where $\delta_{n, m}$ is the Kronecker delta.

In such a situation, $\left\{B_{n}\right\}_{n \geq 0}$ is said to be the monic orthogonal polynomial sequence (MOPS) with respect to $\mathcal{U}$. The MOPS $\left\{B_{n}\right\}_{n \geq 0}$ satisfies a three-term recurrence relation (TTRR):

$$
\left\{\begin{array}{l}
B_{-1}(x)=0, \\
B_{0}(x)=1, \\
B_{n+1}(x)=\left(x-\beta_{n}\right) B_{n}(x)-\gamma_{n} B_{n-1}(x), \quad n \geq 0,
\end{array}\right.
$$

where $\beta_{n} \in \mathbb{C}$ and $\gamma_{n} \in \mathbb{C}^{*}$ for every integer $n \geq 0$ and with $\gamma_{0}=(\mathscr{U})_{0}$. Furthermore, we need the following formula:

$$
\begin{aligned}
\beta_{n} & =\frac{\left\langle\mathcal{U}, x B_{n}^{2}\right\rangle}{\left\langle\mathcal{U}, B_{n}^{2}\right\rangle}, \quad n \geq 0, \\
\gamma_{n+1} & =\frac{\left\langle\mathcal{U}, B_{n+1}^{2}\right\rangle}{\left\langle\mathcal{U}, B_{n}^{2}\right\rangle}, \quad n \geq 0, \\
\left\langle\mathcal{U}, B_{n}^{2}\right\rangle & =\prod_{\nu=0}^{n} \gamma_{v}, \quad n \geq 0,\left(\gamma_{0}=(\mathcal{U})_{0}\right) .
\end{aligned}
$$

When $\mathscr{U}$ is quasi-definite and $A \in \mathbb{P}$ such that $A \mathscr{U}=0$, then $A=0$.

A linear functional $\mathcal{U}$ is said to be positive-definite, if it satisfies (3) with $r_{n}>0$, for every integer $n \geq 0$.

The quasi-definiteness of a linear functional $\mathcal{U}$ is equivalent to (see $[9,10])$

$$
\Delta_{n}(\mathcal{U}):=\left|\begin{array}{ccccc}
(\mathcal{U})_{0} & (\mathcal{U})_{1} & (\mathcal{U})_{2} & \ldots & (\mathcal{U})_{n} \\
(\mathcal{U})_{1} & (\mathcal{U})_{2} & (\mathscr{U})_{3} & \ldots & (\mathcal{U})_{n+1} \\
(\mathcal{U})_{2} & (\mathcal{U})_{3} & (\mathscr{U})_{4} & \ldots & (\mathscr{U})_{n+2} \\
\vdots & \vdots & \vdots & & \vdots \\
(\mathcal{U})_{n} & (\mathscr{U})_{n+1} & (\mathcal{U})_{n+2} & \ldots & (\mathcal{U})_{2 n}
\end{array}\right| \neq 0, \quad \forall n \in \mathbb{N}_{0},
$$

where $\Delta_{n}(\mathscr{U})$ is the Hankel determinant of order $n+1$ of $\mathscr{U}$.

In this way, we have the following formula [5]:

$$
\left\langle\mathscr{U}, B_{n}^{2}\right\rangle=\frac{\Delta_{n}(\mathscr{U})}{\Delta_{n-1}(\mathscr{U})}, \quad n \geq 0,\left(\Delta_{-1}(\mathcal{U})=1\right) .
$$

As a consequence, $\mathcal{U}$ is positive-definite if and only if $\Delta_{n}(\mathcal{U})>0$, for every integer $n \geq 0$.

Lemma 1 (see [8]). Let $\left\{B_{n}\right\}_{n \geq 0}$ be a MOPS with respect to the linear functional $U$ and let $A$ be a monic polynomial with $\operatorname{deg} A=t$. The following statements are equivalent:

(i) The linear functional AU is quasi-definite.

(ii) There exists a MPS $\left\{Q_{n}\right\}_{n \geq 0}$ and parameters $\lambda_{n, v}$ satisfying

$$
\begin{aligned}
A Q_{n}(x) & =\sum_{\nu=n}^{n+t} \lambda_{n, \nu} B_{\nu}(x), \quad n \geq 0, \\
\lambda_{n, n} & \neq 0, \quad n \geq 0 .
\end{aligned}
$$

In this case, $\left\{Q_{n}\right\}_{n \geq 0}$ is the MOPS with respect to $A \mathscr{U}$.

The Hadamard product of two $n \times n$ matrices $A=\left(a_{i j}\right)$ and $\left(b_{i j}\right)$ is defined by $A \circ B \equiv\left(a_{i j} b_{i j}\right)$. The Hadamard product arises in a wide variety of ways. It was perhaps the first significant result published about the Hadamard product that the class of positive semidefinite matrices of a given size is closed under the Hadamard product. For more information, the authors may refer to [11]. Oppenheim in [12] proved the following:

Lemma 2 (see [12]). Let $A=\left(a_{i j}\right)$ and $B=\left(a_{i j}\right)$ be two $n \times n$ positive semidefinite matrices. Then,

$$
\operatorname{det}(A \circ B) \geq \operatorname{det} A \prod_{i=1}^{n} b_{i i} .
$$

\section{Main Results}

For any $(\mu, c) \in \mathbb{C}^{2}$ with $\mu \neq-n, n \geq 0$ and $\mathscr{U} \in \mathbb{P}^{\prime}$, let us consider the linear functional $\mathscr{U}(\mu, c)$ where its moments with respect to the MPS $\left\{(x-c)^{n}\right\}_{n \geq 0}$ that we denote by $(\mathcal{U}(\mu, c))_{n, c}, n \geq 0$, are given by

$$
(\mathcal{U}(\mu, c))_{n, c}=\frac{\mu}{n+\mu}(\mathcal{U})_{n, c}, \quad n \geq 0 .
$$

Equivalently, $\mathscr{U}(\mu, c)$ satisfies the distributional equation:

$$
(\mu-1) \mathcal{U}(\mu, c)-(x-c)(\mathcal{U}(\mu, c))^{\prime}=\mu \mathcal{U} .
$$


3.1. Preservation of the Positive-Definite Property. Assuming that $\mathcal{U}$ is positive definite, our purpose is to find the necessary and sufficient condition on the real parameter $\mu$, in order that the linear functional $\mathcal{U}(\mu, c)$ to be also positive definite.

Theorem 1. Let $\mu$ be a real number with $\mu \neq-n, n \geq 0$, let $c \in \mathbb{R}$, and let $\mathcal{U}$ be a positive-definite linear functional. The following statements are equivalent:

(i) $\mu \in] 0,+\infty[$. (ii) the linear functional $\mathscr{U}(\mu, c)$ satisfying

$$
\begin{aligned}
& (\mu-1) \mathcal{U}(\mu, c)-(x-c)(\mathscr{U}(\mu, c))^{\prime} \\
& =\mu \mathscr{U} \text { is positive definite. }
\end{aligned}
$$

Proof. Taking (10) into account, we can always decompose the determinant of Hankel associate to linear functional $\mathcal{U}(\mu, c)$ thanks to Hadamard's product as follows:

$$
\Delta_{n}(\mathscr{U}(\mu, c)):=\left|\begin{array}{ccccc}
(\mathcal{U}(\mu, c))_{0, c} & (\mathcal{U}(\mu, c))_{1, c} & (\mathcal{U}(\mu, c))_{2, c} & \ldots & (\mathcal{U}(\mu, c))_{n, c} \\
(\mathcal{U}(\mu, c))_{1, c} & (\mathcal{U}(\mu, c))_{2, c} & (\mathcal{U}(\mu, c))_{3, c} & \ldots & (\mathcal{U}(\mu, c))_{n+1, c} \\
(\mathcal{U}(\mu, c))_{2, c} & (\mathcal{U}(\mu, c))_{3, c} & (\mathcal{U}(\mu, c))_{4, c} & \ldots & (\mathcal{U}(\mu, c))_{n+2, c} \\
\vdots & \vdots & \vdots & & \vdots \\
(\mathcal{U}(\mu, c))_{n, c} & (\mathcal{U}(\mu, c))_{n+1, c} & (\mathcal{U}(\mu, c))_{n+2, c} & \ldots & (\mathcal{U}(\mu, c))_{2 n, c}
\end{array}\right|
$$

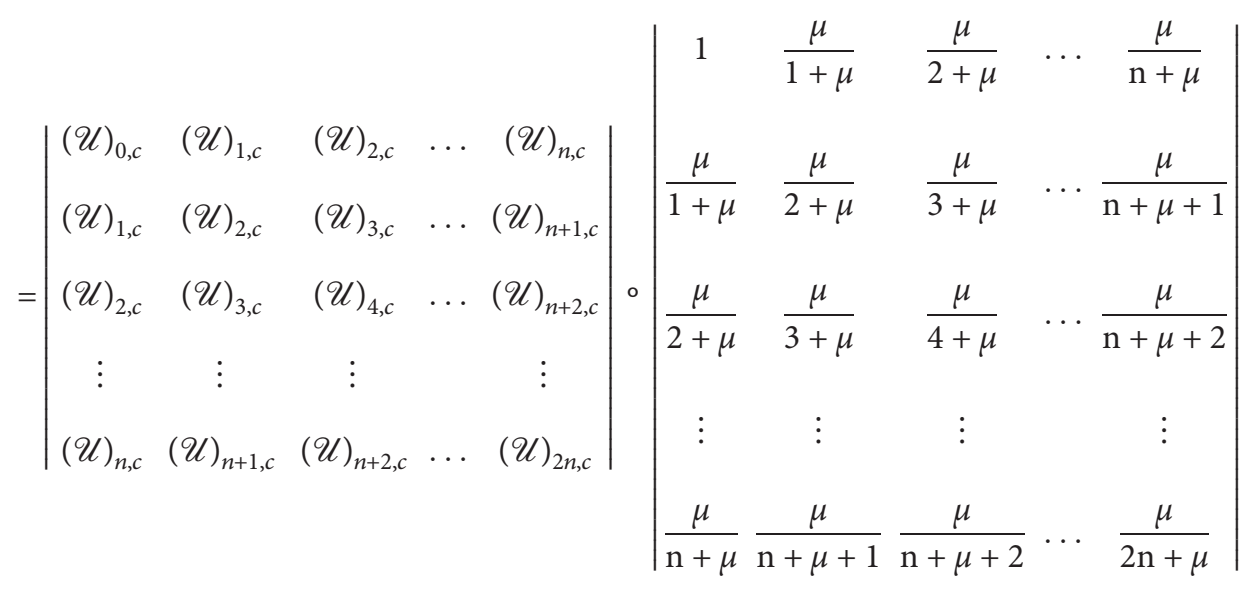

Using Lemma 2 (Oppenheim's inequality) and $\Delta_{n}(\mathcal{U})>0$, for every integer $n \geq 0$ ( $\mathcal{U}$ be a positive-definite linear functional), we find $\prod_{k=0}^{n}(\mu / 2 k+\mu)>0$, if and only if $\mu>0$. Hence, the required result is as follows.

3.2. Classical Case. A quasi-definite linear functional $\mathscr{W}$ is said to be classical if it satisfies a functional equation (Pearson equation):

$$
(\phi \mathscr{W})^{\prime}+\psi \mathscr{W}=0
$$

where $\phi$ and $\psi$ are polynomials such that $\phi$ is monic, $\operatorname{deg} \phi=t \leq 2$, and $\operatorname{deg} \psi=1$.

The corresponding $\left\{B_{n}\right\}_{n \geq 0}$ is said to be classical (for more details, see $[8,13]$ and the literature therein). The classical character of a linear functional is invariant by shifting. Indeed, if $\mathscr{W}$ is a classical linear functional satisfying (10), then for any pair $(a, b) \in \mathbb{C}^{2}$ with $a \neq 0$, the shifted linear functional $\widetilde{\mathscr{W}}=\left(h_{a^{-1}} \circ \tau_{-b}\right) \mathscr{W}$ is also classical and satisfies $(\widetilde{\phi} \widetilde{\mathscr{W}})^{\prime}+\widetilde{\psi} \widetilde{\mathscr{W}}=0$ with $\widetilde{\phi}(x)=a^{-t} \phi(a x+b)$ and $\widetilde{\psi}(x)=a^{1-t} \psi(a x+b)$, where $t=\operatorname{deg} \phi$. We find in [8] a description of the classical linear functional (Hermite, Laguerre, Bessel, and Jacobi).

Proposition 1. If $\mathscr{W}$ is a classical linear functional with $\operatorname{deg} \phi=1$, then

$$
\left(\mu^{-1}(\psi+\mu) \mathscr{W}\right)(\mu, c)=\mathscr{W} .
$$

Proof. $\operatorname{deg} \phi=1$. So, we can write $\phi(x)=x-c$. Let $\mu \in \mathbb{C}$ with $\mu \neq-n, n \in \mathbb{N}$. From (14), it follows that

$$
-(x-c) \mathscr{W}^{\prime}+(\mu-1) \mathscr{W}=(\psi+\mu) \mathscr{W} .
$$
has

Clearly, we have $((\psi+\mu) \mathscr{W})_{0, c}=\mu \neq 0$. Accordingly, one

$$
\left(\mu^{-1}(\psi+\mu) \mathscr{W}\right)(\mu, c)=\mathscr{W} .
$$

Now, let us study the particular situation: 
Namely, $\quad c=0, \quad \mu=\alpha+1, \quad \psi(x)=x-\alpha-1, \quad$ and $\mathscr{W}=\mathscr{L}^{\alpha}$. The Laguerre linear functional with parameter $\alpha \neq-n, n \geq 1$, which is the unique linear functional satisfying (see [8])

$$
\left(x \mathscr{L}^{\alpha}\right)^{\prime}+(x-\alpha-1) \mathscr{L}^{\alpha}=0 .
$$

Since $(\alpha+1)^{-1} x \mathscr{L}^{\alpha}=\mathscr{L}^{\alpha+1}$ and $\alpha \mathscr{L}^{\alpha}-x\left(\mathscr{L}^{\alpha}\right)^{\prime}=$ $(\alpha+1) \mathscr{L}^{\alpha+1}$, accordingly we obtain

$$
\mathscr{L}^{\alpha+1}(\alpha+1,0)=\mathscr{L}^{\alpha} .
$$

The MOPS $\left\{\widehat{L}_{n}^{\alpha}(x)\right\}_{n \geq 0}$ of Laguerre satisfies the first and second structure relations $[6,8]$ :

$$
\begin{gathered}
x\left(\widehat{L}_{n}^{\alpha}\right)^{\prime}(x)=n \widehat{L}_{n}^{\alpha+1}(x)+n(n+\alpha) \widehat{L}_{n-1}^{\alpha}(x), \quad n \geq 0, \\
\widehat{L}_{n}^{\alpha}(x)=\widehat{L}_{n}^{\alpha+1}(x)+n \widehat{L}_{n-1}^{\alpha+1}(x), \quad n \geq 0 .
\end{gathered}
$$

Corollary 1. The polynomials $\widehat{L}_{n}^{\alpha}(x)$ and $\widehat{L}_{n}^{\alpha+1}(x)$ satisfy the following connection relation:

$$
x\left(\widehat{L}_{n}^{\alpha+1}\right)^{\prime}(x)+(\alpha+1) \widehat{L}_{n}^{\alpha+1}(x)=(n+\alpha+1) \widehat{L}_{n}^{\alpha}(x), \quad n \geq 0 .
$$

Proof. Using (20) and (21) and by taking (19) into account,

$$
\begin{aligned}
& x\left(\widehat{L}_{n}^{\alpha+1}\right)^{\prime}(x)+(\alpha+1) \widehat{L}_{n}^{\alpha+1}(x) \\
& =(n+\alpha+1)\left(\widehat{L}_{n}^{\alpha+1}(x)+n \widehat{L}_{n-1}^{\alpha+1}(x)\right), \\
& =(n+\alpha+1) \widehat{L}_{n}^{\alpha}(x), \quad n \geq 0 .
\end{aligned}
$$

Proposition 2. If $\mathscr{W}$ is a classical linear functional with $\operatorname{deg} \phi=2$, then

$$
\left(\mu^{-1} \rho \mathscr{W}\right)(\mu, c)=\theta_{c}(\phi) \mathscr{W} .
$$

Proof. $\operatorname{deg} \phi=2$ and $s=\operatorname{deg} \phi-2=\operatorname{deg} \psi-1$. Let $c$ be a zero of $\phi$. From the Euclidean division of $\psi$ by $\theta_{c}(\phi)$, we always have

$$
\psi(x)=-\mu \theta_{c}(\phi)(x)+\rho(x),
$$

where $\rho \in \mathbb{P}$ with $\operatorname{deg} \rho \leq \operatorname{deg}(\psi)-1$. Clearly, $\mu$ is given by

$$
\mu=-\frac{\psi^{(s+1)}(0)}{(s+1) !} \neq 0 .
$$

Since $\operatorname{deg} \psi=\operatorname{deg} \phi-1$, then $\mu$ is the leading coefficient of $-\psi$.

From (14) and (25), we can write

$$
(\mu-1)\left(\theta_{c}(\phi) \mathscr{W}\right)-(x-c)\left(\theta_{c}(\phi) \mathscr{W}\right)^{\prime}=\rho \mathscr{W} .
$$

Notice that $\operatorname{deg} \rho \geq 0$, otherwise the linear functional $\theta_{c}(\phi) \mathscr{W}$ will satisfy $(\mu-1)\left(\theta_{c}(\phi) \mathscr{W}\right)-(x-c)\left(\theta_{c}(\phi) \mathscr{W}\right)^{\prime}=$ 0 and then its moments with respect to the MPS $\left\{(x-c)^{n}\right\}_{n \geq 0}$ are such that $(n+\mu)\left(\theta_{c}(\phi) \mathscr{W}\right)_{n, c}=0, \quad n \geq 0$. So, there exists an integer $n_{0} \geq 0$ such that $\left(\theta_{c}(\phi) \mathscr{W}\right)_{n, c}=0, n \geq n_{0}$, i.e., $\quad(x-c)^{n_{0}} \theta_{c}(\phi) \mathscr{W}=0 \quad$ which contradicts the quasi-definiteness of $\mathscr{W}$.

Directly, we have

$$
\left(\mu^{-1} \rho \mathscr{W}\right)(\mu, c)=\theta_{c}(\phi) \mathscr{W} .
$$

Now, let us study some particular situations.

$I_{1} \quad$ (Bessel case): namely, $\quad c=0, \quad \mu=2 \alpha, \quad \psi(x)=$ $-2(\alpha x+1)$, and $\mathscr{W}=\mathscr{B}^{\alpha}$. The Bessel quasi-definite linear functional with parameter $\alpha \neq-(n / 2), n \geq 0$, which is the unique monic linear functional satisfying

$$
\left(x^{2} \mathscr{B}^{\alpha}\right)^{\prime}-2(\alpha x+1) \mathscr{B}^{\alpha}=0 \text {. }
$$

We can easily show that

$$
-\alpha x \mathscr{B}^{\alpha}=\mathscr{B}^{\alpha+(1 / 2)} \text {. }
$$

It follows that

$$
(2 \alpha-1) \mathscr{B}^{\alpha+(1 / 2)}-x\left(\mathscr{B}^{\alpha+(1 / 2)}\right)^{\prime}=2 \alpha \mathscr{B}^{\alpha},
$$

so that

$$
\mathscr{B}^{\alpha}(2 \alpha, 0)=\mathscr{B}^{\alpha+(1 / 2)} .
$$

Denote by $\left\{\widehat{B}_{n}^{(\alpha)}(x)\right\}_{n \geq 0}$ the MOPS with respect to the Bessel linear functional $\mathscr{B}^{\alpha}$. The MOPS $\left\{\widehat{B}_{n}^{\alpha}(x)\right\}_{n \geq 0}$ satisfies the first structure relation [8]:

$$
x^{2}\left(\widehat{B}_{n+1}^{(\alpha)}\right)^{\prime}(x)=(n+1)\left(x-\frac{1}{n+\alpha}\right) \widehat{B}_{n+1}^{(\alpha)}(x)-\delta_{n} \widehat{B}_{n}^{(\alpha)}, \quad n \geq 0,
$$

where

$$
\delta_{n}=\frac{(n+1)(n+2 \alpha-1)}{(2 n+2 \alpha-1)(n+\alpha)^{2}}, \quad n \geq 0 .
$$

Corollary 2. The polynomials $\widehat{B}_{n+1}^{(\alpha)}(x)$ and $\widehat{B}_{n}^{(\alpha+(1 / 2))}(x)$ satisfy the following relation:

$$
x\left(\widehat{B}_{n+1}^{(\alpha)}\right)^{\prime}(x)-(n+1) \widehat{B}_{n+1}^{(\alpha)}(x)=-\left(\frac{n+1}{n+\alpha}\right) \widehat{B}_{n}^{(\alpha+(1 / 2))}(x), \quad n \geq 0 .
$$

Proof. Using (33), we obtain for any integer $n \geq 0$

$$
x Q_{n}(x)=\widehat{B}_{n+1}^{(\alpha)}(x)-\frac{(n+2 \alpha-1)}{(2 n+2 \alpha-1)(n+\alpha)} \widehat{B}_{n}^{(\alpha)}(x), \quad n \geq 0,
$$

where $Q_{n}(x)=-(n+1 / n+\alpha)\left(x\left(\widehat{B}_{n+1}^{(\alpha)}\right)^{\prime}(x)-(n+1) \widehat{B}_{n+1}^{(\alpha)}\right.$ $(x)), n \geq 0$.

By Lemma 1 , with $A(x)=x, \quad B_{n}=\widehat{B}_{n}^{(\alpha)}$, and $\lambda_{n, n}=-((n+2 \alpha-1) /(2 n+2 \alpha-1)(n+\alpha)), n \geq 0, \quad\left(\lambda_{0,0}=\right.$ $-(1 / \alpha))$ and by taking (22) and (24) into account, the MPS $\left\{Q_{n}(x)\right\}_{n \geq 0}$ is orthogonal with respect to the linear functional $\mathscr{B}^{(\alpha+(1 / 2))}$. Thus, $Q_{n}(x)=\widehat{B}_{n}^{(\alpha+(1 / 2))}(x), n \geq 0$. This leads to (35).

$I_{2} \quad$ (Jacobi case): namely, $\quad c=1, \quad \mu=\alpha+\beta+2$, $\psi(x)=-(\alpha+\beta+2) x+\alpha-\beta$, and $\mathscr{W}=\mathscr{J}^{\alpha, \beta}$. The Jacobi 
quasi-definite linear functional with parameters $\alpha$ and $\beta$ where $\alpha, \beta \neq-n$ and $\alpha+\beta \neq-n-1, n \geq 1$, which is the unique monic linear functional satisfying

$$
\left(\left(x^{2}-1\right) \mathscr{J}^{(\alpha, \beta)}\right)^{\prime}+(-(\alpha+\beta+2) x+\alpha-\beta) \mathscr{J}^{(\alpha, \beta)}=0 .
$$

Since

$$
\frac{\alpha+\beta+2}{2(\alpha+1)}(x+1) \mathscr{J}^{(\alpha, \beta)}=\mathscr{J}^{(\alpha+1, \beta)},
$$

it follows that

$$
(\alpha+\beta+1) \mathscr{J}^{(\alpha+1, \beta)}-(x-1)\left(\mathscr{J}^{(\alpha+1, \beta)}\right)^{\prime}=(\alpha+\beta+2) \mathscr{J}^{(\alpha, \beta)},
$$

So, we can deduce that

$$
\mathscr{J}^{(\alpha, \beta)}(\alpha+\beta+2,0)=\mathscr{J}^{(\alpha+1, \beta)} .
$$

Denote by $\left\{\widehat{J}_{n}^{(\alpha, \beta)}(x)\right\}_{n \geq 0}$ the MOPS with respect to the Jacobi linear functional $\mathscr{J}^{(\alpha, \beta)}$. The MOPS $\left\{\widehat{J}_{n}^{(\alpha, \beta)}(x)\right\}_{n \geq 0}$ satisfies the first structure relation for $n \geq 0$ [8]:

$$
\begin{aligned}
\left(x^{2}-1\right)\left(\widehat{J}_{n+1}^{(\alpha, \beta)}\right)^{\prime}(x)= & (n+1)\left(x+\frac{\alpha-\beta}{2 n+\alpha+\beta+2}\right) \widehat{J}_{n+1}^{(\alpha, \beta)}(x) \\
& -(2 n+\alpha+\beta+3) \gamma_{n+1} \widehat{J}_{n}^{(\alpha, \beta)},
\end{aligned}
$$

where

$$
\gamma_{n+1}=\frac{4(n+1)(n+\alpha+\beta+1)(n+\alpha+1)(n+\beta+1)}{(2 n+\alpha+\beta+1)(2 n+\alpha+\beta)^{2}(2 n+\alpha+\beta+3)}, \quad n \geq 0 .
$$

Corollary 3. The polynomials $\widehat{J}_{n+1}^{(\alpha, \beta)}(x)$ and $\widehat{J}_{n}^{(\alpha+1, \beta)}(x)$ satisfy the following relation:

$$
(x-1)\left(\widehat{J}_{n+1}^{(\alpha, \beta)}\right)^{\prime}(x)-(n+1) \widehat{J}_{n+1}^{(\alpha, \beta)}(x)=\varepsilon_{n} \widehat{J}_{n}^{(\alpha+1, \beta)}(x),
$$

where for any integer $n \geq 0$,

$$
\varepsilon_{n}=-2 \frac{(n+1)(n+\beta+1)}{2 n+\alpha+\beta+2}, \quad n \geq 0
$$

Proof. Using (41), we obtain for any integer $n \geq 0$,

$$
\begin{aligned}
(x+1) Q_{n}(x)= & \widehat{J}_{n+1}^{(\alpha, \beta)}(x) \\
& +\frac{2(n+\alpha+1)(n+\alpha+\beta+1)}{(2 n+\alpha+\beta+1)(2 n+\alpha+\beta+2)} \widehat{J}_{n}^{(\alpha, \beta)}(x), \\
& n \geq 0,
\end{aligned}
$$

where

$$
\begin{aligned}
Q_{n}(x)= & -\frac{2 n+\alpha+\beta+2}{2(n+1)(n+\beta+1)} \\
& \cdot\left((x-1)\left(\widehat{J}_{n+1}^{(\alpha, \beta)}\right)^{\prime}(x)-(n+1) \widehat{J}_{n+1}^{(\alpha, \beta)}(x)\right), \quad n \geq 0 .
\end{aligned}
$$

By Lemma 1 , with $A(x)=x+1, \quad B_{n}=\widehat{J}_{n}^{(\alpha, \beta)}$, $\lambda_{n, n}=(2(n+\alpha+1)(n+\alpha+\beta+1) /(2 n+\alpha+\beta+1)(2 n+\alpha+$ $\beta+2)) \neq 0, n \geq 0$, and by taking (38) and (40) into account, xthe MPS $\left\{Q_{n}(x)\right\}_{n \geq 0}$ is orthogonal with respect the linear functional $\mathscr{J}^{(\alpha+1, \beta)}$. Thus, $Q_{n}(x)=\widehat{J}_{n}^{(\alpha+1, \beta)}(x), n \geq 0$. This leads to (43).

\section{Data Availability}

All data required for this paper are included within this paper.

\section{Conflicts of Interest}

The authors declare that they have no conflicts of interest.

\section{Acknowledgments}

The authors extend their appreciation to the Deanship of Scientific Research at Majmaah University for funding this work under project number RGP-2019-5.

\section{References}

[1] B. Aloui, "Chebyshev polynomials of the second kind via raising operator preserving the orthogonality," Periodica Mathematica Hungarica, vol. 76, no. 1, pp. 126-132, 2018.

[2] B. Aloui, "Characterization of Laguerre polynomials as orthogonal polynomials connected by the Laguerre degree raising shift operator," The Ramanujan Journal, vol. 45, no. 2, pp. 475-481, 2018.

[3] G. Bennett, "Hausdorff means and moment sequences," Positivity, vol. 15, no. 1, pp. 17-48, 2011.

[4] X. Chen, H. Liang, and Y. Wang, "Total positivity of Riordan arrays," European Journal of Combinatorics, vol. 46, pp. 68-74, 2015.

[5] T. S. Chihara, An Introduction to Orthogonal Polynomials, Gordon \& Breach, New York, NY, USA, 1978.

[6] R. Sfaxi, "On inverse problem leading to second-order linear functionals," Journal of Approximation Theory, vol. 162, no. 4, pp. 678-686, 2010.

[7] W. Chammam, "A new discrete representation of linear functionals," Iranian Journal of Science and Technology, Transactions A: Science, vol. 43, no. 4, pp. 1829-1833, 2019.

[8] P. Maroni, "Une théorie algébrique des polynômes orthogonaux. Application aux polynômes orthogonaux semiclassiques," in Orthogonal Polynomials and their Applications, IMACS Annals Computing and Applied Mathematic, C. Brezinski, Ed., vol. 9, pp. 95-130, Baltzer, Basel, Switzerland, 1991.

[9] W. Chammam, "Generalized harmonic numbers, Jacobi numbers and a Hankel determinant evaluation," Integral Transforms Spec. Funct.vol. 30, no. 2, pp. 581-563, 2019.

[10] W. Chammam, F. Marcellán, and R. Sfaxi, "Orthogonal polynomials, Catalan numbers, and a general Hankel 
determinant evaluation," Linear Algebra and Its Applications, vol. 436, no. 7, pp. 2105-2116, 2012.

[11] X.-D. Zhang and C.-X. Ding, "The equality cases for the inequalities of Oppenheim and Schur for positive semi-definite matrices," Czechoslovak Mathematical Journal, vol. 59, no. 1, pp. 197-206, 2009.

[12] A. Oppenheim, "Inequalities connected with definite Hermitian forms," Journal of the London Mathematical Society, vol. s1-5, no. 2, pp. 114-119, 1930.

[13] P. Maroni, "Fonctions eulériennes. Polynômes orthogonaux classiques," Techniques de l'ingénieur, vol. 154, pp. 1-30, 1994. 